\title{
Das Fremde.
}

\section{Anmerkungen zu seinem Auftritt in Kultur und Wissenschaft}

\author{
Alexander Honold
}

\section{Der Ort des Fremden}

Auf den ersten Blick scheinen sich die Rückzugsgebiete des Fremden dramatisch verringert zu haben. Keine weißen Flecken mehr weist die Weltkarte auf, von dunklen Kontinenten keine Rede. Und dennoch gab es noch nie so viel an imaginierter und auch tatsächlich erfahrbarer Fremdheit, wie ausgerechnet im Zeitalter einer scheinbar grenzenlosen, uniformen Verwestlichung. In der Epoche seit Ende des Zweiten Weltkriegs haben exponentiell ansteigende weltweite Reiseaktivitäten, Migrationsbewegungen und Kommunikationsnetze das Fremde überall aufgespürt, entzaubert oder vertrieben. Zugleich aber wachsen die Begegnungen mit Erscheinungsformen kultureller Fremdheit derart, dass Kultur selbst inzwischen, und mit durchaus guten Gründen, als ein synkretistischer Modus der Ermöglichung und Verarbeitung von Fremdkontakten verstanden werden kann, so jedenfalls die These des postkolonialen Theoretikers Homi Bhabha. ${ }^{1}$ Nicht zufällig vollzog sich der Eintritt in das Zeitalter globaler Mobilität zeitgleich mit den teils sprunghaften, teils allmählichen Prozessen der Entkolonialisierung, von denen unterschiedlichste Weltregionen, Nationen und Akteure in mehre-

1. Der postkoloniale Theoretiker Homi Bhabha legt Wert darauf, den Ort des Kulturellen selbst als Zwischenzone oder Übergangsraum differenter Herkünfte, Ethnien, Lebensweisen zu denken; so gesehen haftet den Konzepten von Inter- oder Multikulturalität eine gewisse Redundanz an (Homi K. Bhabha: The location of culture, London, New York: Routledge 1994; deutsch: Die Verortung der Kultur, Tübingen: Stauffenburg 2000). 
ren Schüben erfasst wurden. Mit der Aufkündigung manifester Kolonialverhältnisse wurden die sie flankierenden politischen Machtstrukturen in die Latenz zurückgedrängt, ökonomische Abhängigkeiten dagegen noch deutlicher sichtbar.

Definitivbeseitigt hat die globalisierte Weltordnung sowohl die Rollenfiktion des unbeteiligten Beobachters wie die des neutralen Schiedsrichters oder fairen Maklers. Nach dem Ende der Ost-WestSpaltung und ihrer binären Logik der Systemkonkurrenz erweist sich: Zur schönen neuen Welt und ihren global players existiert kein Außen mehr. Der Andere, der Fremde wird zum kontingenten Merkmalsträger einer relationalen (und jeweils relativierbaren) Binnendifferenz. Im world wide web eines ubiquitären westlichen Lebensstils, so die Befürchtungen, droht eine nie gekannte Nivellierung regionaler Eigenarten und kultureller Diversität. ${ }^{2}$ In diesem Räsonnement äußert sich ein wachsendes Bedürfnis nach Distinktion und Differenz, das aus den gesteigerten Aufmerksamkeitswerten für alle Zeichen des Fremden immer neue Anreize bezieht; in den Künsten und Wissenschaften, auf dem Markt der Güter und Lebensstile. $^{3}$ Oft erzeugen die interkulturellen Vergleichsmöglichkeiten erst jene Unterschiede, die sie vermeintlich einzuebnen im Begriff stehen. Paradox formuliert, bemisst sich die zeitgemäße Instrumentalisierung des Fremden just daran, wie sehr es auch ästhetisch zu dem geworden ist, was es der Sache nach immer schon war: Synonym des Unverfügbaren.

Das Fremde ist die kulturelle Ressource der Moderne schlechthin. An definitorischen Festschreibungen kultureller Identität herrschte im vergangenen Jahrhundert wahrlich kein Mangel, gerade angesichts ihrer wachsenden Instabilität. Denn ob diese sunverbrüchlichen` Wesenheiten nun religiös, ethnisch, nationalgeschichtlich oder ökonomisch konturiert wurden, das Verbindende und Verlässliche an ihnen war einzig die mit immer kürzeren Halbwertszeiten gewahrte Grenzziehung gegenüber dem Fremden. Das Zusammenspiel von Inklusion und Exklusion, von Selbstaffirmation und Krisenerfahrung verdankt seine Energie nicht den reklamierten Positionen, sondern dem dynamischen, kaum fixierbaren Ort der kul-

2. Das Spannungsfeld von Globalisierung und »culturalism«, verstanden als »conscious mobilization of cultural differences in the service of a larger national or transnational politics«, untersucht Arjun Appadurai: Modernity at large. Cultural Dimensions of Globalisation, Minneapolis: Univ. of Minnesota Press 1996, S. 15.

3. »Nicht die Fremden, sondern der Mangel an Fremdheit, nicht die Anderen, sondern die Nivellierung der Alterität sind bedrohlich« (Jochen K. Schütze: Vom Fremden, Wien: Passagen-Verlag 2000, S. 18f). 
turellen Differenz. Modernität, nicht im epochalen, sondern im prozessualen Sinne gefasst, bedeutet Ent-Selbstverständlichung.

Weiter zurückgreifend, ist die gesamte Geschichte der Neuzeit snach Kolumbus durch die Bedeutung des Kontaktes mit fremden Welten markiert. Das Neue sentdecken wänder anlegen sind eins. Die Ambivalenz dieses epistemologischen Einschnitts haben Tzvetan Todorov ${ }^{4}$ und Stephen Greenblatt ${ }^{5}$ auf neue Weise hervortreten lassen; mit ihren Studien über die Landnahme durch Kolumbus und Cortez begann, auch im Hinblick auf das zwiespältige Datum des fünfhundertjährigen Amerika-Jubiläums, ein Neuaufschwung kulturwissenschaftlicher Fremdeforschung am Ausgang des 20. Jahrhunderts. Zwei nachhaltige Erschütterungen hatte das Zeitalter der Entdeckungen zur Folge: da war einerseits der ethnographische Schock über die verschobenen Grenzen des Menschseins, zum anderen die expansive Dynamik des europäischen Kolonialismus. Von seinen abenteuerlichen und gewaltsamen Übergriffen auf überseeische Gebiete und ihre Bewohner blieb auch Westeuropa selbst nicht unberührt. In Daniel Defoes Robinson Crusoe von 1719, einem Grundbuch der neuzeitlichen Globalisierung, gibt es eine Schlüsselszene des tiefsten, bebenden Entsetzens. Es ist der Moment, in dem der gestrandete Selbsthelfer Robinson nach Jahrzehnten der Einsiedlerexistenz auf seiner Insel im Südatlantik feststellen muss, nicht allein zu sein. Er bewegte sich auf dem vermeintlich einsamen Eiland als selbstverständlicher Herr im Hause - bis ihn eines Tages die Entdeckung einer fremden menschlichen Fußspur eines anderen belehrt. Die nachgerade erstaunliche Fiktion des europäischen Kolonialismus, allein auf der Welt zu sein, findet in diesem Augenblick tiefsten Erschreckens ihren bündigen Ausdruck. Ihm antwortet, Jahrhunderte später, das Entsetzen über die Auswüchse des europäischen Blutrauschs: »The horror, the horror « - mit diesen letzten Worten in Heart of Darkness Joseph Conrads endet die vermeintliche Kulturmission des ins tiefste Afrika verstrickten Neu-Barbaren Kurtz. Nun aber rührt das Grauen von der grenzenlosen Gewaltherrschaft des unheimlichen weißen Mannes.

Mit dem eurozentrischen Blick auf den sogenannten >Rest ‘ der Welt wurden freilich auch Erfahrungen angebahnt, die solcher Hybris ein Ende bereiteten. Im postkolonialen Zeitalter sind die Be-

4. Tzvetan Todorov: Die Eroberung Amerikas. Das Problem des Anderen, Frankfurt/M.: Suhrkamp 1985.

5. Stephen Greenblatt: Wunderbare Besitztümer. Die Erfindung des Fremden: Reisende und Entdecker, Berlin: Wagenbach 1994. 
schriftung und Bebilderung des Fremden politisch umkehrbar geworden durch die counter-Strategien des writing back und viewing back. Doch fungieren 'Entfremdung` und 'Verfremdung ‘ bereits als diagnostische oder programmatische Schlagworte auf der Agenda der Moderne. Es ist der Blick in den Spiegel, der Alteritätskrisen produziert. In den Zeichenordnungen des 20. Jahrhunderts hat die kulturelle Produktion solche inneren Risse und Spaltungen immer wieder entstehen lassen und zu reflektieren gewusst, mit denen es einer Gesellschaft gelingt, sich selbst fremd zu werden.

Wer in Kultur und Wissenschaft an der Geschichte des Fremden mitschreibt, ist nicht Erfinder oder Entdecker, sondern lediglich Nutznießer dieser Umstände. Wenn es zutrifft, dass die Phänomene ihre größte Prägnanz im Augenblick des Verschwindens gewinnen, so darf der Fortbestand des Fremden und seiner zahllosen Schattierungen im Diskurs wie in der Lebenswelt bis auf Weiteres als gesichert gelten. Zeigt sich doch die Präsenz des »Kulturthemas Fremdheit « ${ }^{6}$ ebenso omnipräsent wie ungreifbar. Als `Herausforderung beschäftigt das Fremde nicht nur Künstler, Politiker, Sozialarbeiter oder Tourismusexperten, sondern auch akademische Forschungsprogramme. Die Erscheinungsformen und Folgeprobleme des wachsenden interkulturellen Austauschs haben es mit Macht auf die Tagesordnung gesetzt und mit einem höchst ambivalenten Bedeutungshaushalt versehen. Philosophie, Geschichte, Soziologie, Psychologie, letztlich der gesamte Fächerkanon der Sozial- und $\mathrm{Hu}$ manwissenschaften haben sich des Themas angenommen. ${ }^{7}$

Dabei lag der Schwerpunkt zunächst auf der geschichtlichen Nachzeichnung von interkulturellen Kontakten und stereotypen Fremdbildern, die es aus ideologiekritischer Sicht zu hinterfragen und zu korrigieren galt, wobei oft der Eindruck vorherrschte, solche Projektionen seien durch die richtige und authentische Repräsentation des Fremden zu ersetzen. In jüngerer Zeit aber haben die Kulturwissenschaften, angeregt durch die postkoloniale Aufmerksam-

6. Alois Wierlacher (Hg.): Kulturthema Fremdheit. Leitbegriffe und Problemfelder kulturwissenschaftlicher Fremdheitsforschung, München: ludicium 1993.

7. Zur philosophischen Fremdeforschung: Bernhard Waldenfels: Der Stachel des Fremden, Frankfurt/M.: Suhrkamp 1990; ders.: Topographie des Fremden, Frankfurt/M.: Suhrkamp 1997; Hinrich Fink-Eitel: Die Philosophie und die Wilden, Hamburg: Junius 1994; zur Geschichte: Urs Bitterli: Die Wilden und die Zivilisierten, München: Beck 1976; über die soziologischen und politischen Debatten informieren: ULrich Bielefeld (Hg.): Das Eigene und das Fremde, Hamburg: Junius 1991 und Herfried Münkler (Hg.): Furcht und Faszination. Facetten der Fremdheit, Berlin: Akademie Verlag 1997. 
keit für Phänomene der Kreolisierung, Hybridität und métissage, ${ }^{8}$ vermehrt auch die poetische Produktivität bestimmter Figuren des Fremden in den Blick genommen, etwa die Position des Barbaren, die des stigmatisierten Außenseiters oder überhaupt die Rolle des "Dritten «. ${ }^{9}$ Zugleich sind mit der weit über die Fachgrenzen hinaus beachteten Methodendiskussion der Anthropologen und Ethnographen die Probleme der Repräsentation kultureller Differenz in den Brennpunkt des Interesses (und der Kritik) gerückt, ebenso die Fragen einer zunehmenden 'Ethnisierung، kultureller Phänomene, etwa mit der unter strategischen Gesichtspunkten verfolgten Identitätspolitik, wie sie von postkolonialen Theoretikern vorgeschlagen wurde. Scheinbar biologische Faktoren wie Herkunft, Rasse und Geschlecht werden dabei als kulturelle Konstruktionen beschrieben, die einerseits zwar diskriminierend, andererseits aber auch identitätsstiftend wirken können.

»Unterschiedenes ist gut«, so heißt es bereits bei Hölderlin. Diese Devise beschreibt den derzeit letzten Konsens kulturwissenschaftlicher wie politischer Debatten vielleicht am treffendsten. Gemeint ist die Aufkündigung eines hegemonialen Begriffs von Kultur und damit verbunden die Anerkennung, wenn nicht Auszeichnung des Marginalen und Dezentralen. Der aufklärerische Begriff der Toleranz, wie ihn exemplarisch Voltaires Traité sur la tolerance $(1763)^{10}$ zur Selbstverständigung der societas civilis gegen die gewaltsamen Übergriffe eines politisch oder auch ethnisch >Anderen formuliert, trägt den Widerspruch des Universalen und Partikularen

8. Vgl. Horst Turk: »Alienität und Alterität als Schlüsselbegriffe der Kultursemantik«, in: Jahrbuch für Internationale Germanistik, Jg. XII (1990), 1, S. 8-31; Bernd Lenz/Hans-Jürgen Lüsebrink (Hg.): Fremdheitserfahrung und Fremdheitsdarstellung in okzidentalen Kulturen, Passau: Wiss.-Verl. Rothe 1999 und, in kritischer Perspektive, Jeroen Dewulf: »Das Recht auf Hybridität: Über Kreolismus und Anthropophagie in der Literaturwissenschaft«, in: Canadian Review of Comparative Literature, Dezember 2000, S. 611-624.

9. Über die diskursive Konstruktion des Barbaren Michel Henry: Die Barbarei. Eine phänomenologische Kulturkritik, Freiburg [u.a.]: Karl Alber 1994; Manfred Schneider: Der Barbar. Genealogie der Endzeitstimmungen, München: Hanser 1997; zum Stereotyp des Außenseiters in Deutschland Hans Mayer: Außenseiter, Frankfurt/M.: Suhrkamp 1975; Sander L. Gilman: Difference and pathology. Stereotypes of sexuality, race and madness, Ithaca: Cornell Univ. Press 1985; zur Figur des Dritten Claudia Breger/Tobias Döring (Hg): Figuren der/des Dritten. Erkundungen kultureller Zwischenräume, Amsterdam: Rodopi 1998.

10. Zum Toleranz-Konzept und seiner Geschichte vgl. Rolf Kloepfer/Burckhardt Dücker (Hg.): Kritik und Geschichte der Intoleranz, Heidelberg: Synchron 2000. 
in sich, der im Kolonialismus virulent wird. Grenzüberschreitungen sind stets auch Grenzverletzungen. Der Eingriff in die fremde Kultur, in welcher Absicht auch immer, ist stets ein Akt der Gewalt: schöpferisch und zerstörerisch. Am deutlichsten zeigt sich dies in den Menschenrechtsdebatten seit der Aufklärung, die die Möglichkeiten des Menschseins ständig erweitert haben (konstitutionell, moralpolitisch, biologisch), zugleich aber die Souveränität des Rechts immer aufs Neue in Frage stellen. ${ }^{11}$ Die Zuschreibungen des im Multikulturalismus so wünschbaren Toleranzdenkens bleiben wirkungslos, wenn diese doppelte Logik des Konstruktiven und Destruktiven von Interkulturalität nicht stets mit verhandelt wird.

\section{Die Darstellung des Fremden}

Wo Fremdheit zur Sprache kommt, sind zweiwertige, bipolare Ordnungen vorausgesetzt. Nicht nur solche vom Schlage der einfachen, manichäischen Weltaufteilung in Hier und Dort, Nord und Süd, Wir und die Anderen ${ }^{12}$ - auch die zwiespältige Affektstruktur im Gefolge des Phänomens scheint einer solchen zweiwertigen Grammatik unterworfen. Eine symptomatische Verbindung aus Abwehr und Verlangen ist schon aus ältesten Darstellungen fremdartiger Wesen und Regionen zu erkennen; >Furcht und Faszination ‘ oder auch >Faszination und Schrecken des Fremden - solche Wendungen umreißen schematisch ein Spannungspotential, dessen xenophile und xenophobe Linien bis in die Gegenwart hinein nachzuzeichnen wären. Auf der einen Seite obsessive Fremd- oder gar Feindbilder, das Menetekel des Zusammenpralls der Kulturen - auf der anderen die trotzigen oder eher melancholischen Formen, sich gegen das Verschwinden des Fremden aufzubäumen. Auch im Hinblick auf die aus Literaturgeschichte wie Psychoanalyse bekannten Motive des ,Unheimlichen ‘ kann plausibel von einer Angstlust gegenüber dem Fremden gesprochen werden. Befremdlich ist, was sich im Auftreten wie in der Wirkung einer klaren Zuordnung entzieht und deshalb Unruhe hervorruft. Jedoch: Leiden hochkomplexe Gesellschaftsordnungen heutzutage nicht längst, jenseits derartiger psychosozialer

11. Jede Menschenrechtsforderung bringt neue Rechte hervor und damit den Streit über die Interpretations- und Definitionsmacht einer jeden kulturellen Bewegung (dazu Costas Douzinas: The End of Human Rights: Critical Legal Thought at the Fin-de-Siécle, 0xford [u.a.]: Hart 2000).

12. Abdul JanMohamed: Manichean Aesthetics. The Politics of Literature in Colonial Africa, Amherst: Univ. of Massachusetts Press 1983. 
Dispositionen, unter einer grenzenlosen, durch nichts zu erschütternden Gleichgültigkeit ${ }^{13}$

Umso wichtiger wird es dann, den Akzentverschiebungen nachzuspüren. Weil der, die oder das Fremde in dreierlei Gestalt erscheint, kann es trotz ineinander fließenden Sprachgebrauchs sinnvoll sein, die Aspekte ein wenig im Sinne einer historischen Semantik zu sortieren. Die Fremde hat als Abstraktum eine primär räumliche Bedeutung; gemeint ist jeweils eine Region, die aus Sicht der Sprecher wenig bekannt ist und weit entfernt liegt, ${ }^{14}$ ein Gebiet am Rande, auf der anderen Seite oder im feindlichen Lager. Trotz ihrer geographischen und kulturellen Andersheit ist diese Fremde weder unerreichbar noch entzieht sie sich der Darstellbarkeit. Reisepläne und Entdeckungsfahrten zielen in die Fremde, sie kommt als Durchgangsstadium für Wanderjahre und Bildungsromane in Betracht. Ihr Gegenpart ist die Heimat, in der Herkunft und Zuhause in eins fallen. Deren Bild beginnt erst zu leuchten, wenn diese Heimat selbst zur biographischen oder sozialen Fremde geworden ist. Glückliche und öfter noch misslingende Heimkehrergeschichten, von Homers Kriegs- und Fahrensmann Odysseus bis zu den nach Amerika verschlagenen Söhnen bei Gottfried Keller oder Franz Kafka, entfalten zwischen beiden Polen ihre Dramatik. Und sie kosten jenen sentimentalischen Sinngehalt der Fremde aus, der erkennen lässt, welche schwerwiegende Bedeutung dem Überwinden räumlicher Distanzen in früheren Jahrhunderten zukam.

SolangeHeimat und Fremde als räumlich entflochtenes Gefüge intakt bleiben, hat der Fremde den Rang einer Ausnahmeerscheinung. In der Romantik formulierte das Auftauchen des Fremden die negative, desillusionierte Version des Bildungsgedankens. "Fremd bin ich eingezogen, fremd zieh ich wieder aus«, singt zu frostigen Klavierakkorden die Liedstimme des Wanderers in Franz Schuberts Winterreise. Nicht mehr ein bestimmter Raum ist nun Träger der Fremdheit, sondern der Mensch, namentlich der unbehauste, wandernde Geselle, dessen Rollenbild sich nach 1800 von der edukatorischen Zwischenphase zur dauerhaften Existenzweise verschiebt. Permanente Ortsveränderung als solche avanciert nun $\mathrm{zu}$ einer

13. Rudolf Stichweh: »Der Fremde - Zur Soziologie der Indifferenz«, in: H. Münkler (Hg.): Furcht und Faszination, S. 45-61.

14. Die Bedeutungskomponente des räumlich Distanzierten liegt bereits in dem germanischen Wort ıfram vor, das als etymologische Wurzel des Adjektivs ıfremd rekonstruiert wurde und demnach soviel wie sentfernt<, sfern oder auch sfern von bedeutet. (Friedrich Kluge: Etymologisches Wörterbuch der deutschen Sprache, Berlin, New York: De Gruyter 1975, S. 218). 
kulturellen Qualität, die gelegentlich auch einer sozialen Dissidenz Ausdruck verleihen kann. Im Mitteleuropa der Schollenbewohner ging, wie sich etwa an der Konjunktur der >Zigeuner<-Motivik im 19. Jahrhundert ablesen lässt, ${ }^{15}$ das Gespenst eines neuen Nomadentums um, das dann in unserer spätmodernen Zeit so viel Prominenz gewonnen hat. Die armutsbedingte Migration, die Mobilisierung tradierter Formen der Sesshaftigkeit im Zeichen der Massen- und Maschinenarbeit, all die Formen und Symptome aufgelöster Ortsbindungen wurden im späten 19. Jahrhundert zur Quelle sozialer Ängste und Phantasmagorien. Von dieser Lage künden etwa die vielen Amerika-Wanderer und alsbald auch die "Amerikamüden«, deren romanhafte Schicksale den Weg in die literarische Moderne als basso continuo begleiten. ${ }^{16}$

Fremdsein als existenzielle Haltung und kulturkritisches Privileg: Dieses produktive Potenzial der Figur des Unzugehörigen hat Georg Simmel in seiner Skizze über den Fremden herausgestellt. Aus Sicht der sesshaften Mehrheit ist der Fremde ein leibhaftiges Provisorium (der Gast, der bleibt) in dem paradoxen Modus, „daß der Ferne nah ist «. ${ }^{17}$ Er zeigt sich als gewesener Wanderer, »der, obgleich er nicht weitergezogen ist, die Gelöstheit des Kommens und Gehens nicht ganz überwunden hat."Statt der stigmatisierenden Merkmale von Ortsverlust und Entwurzelung aber betont Simmel den Vorteil einer größeren >Beweglichkeit` des Fremden in der Moderne. Gewonnen wird das positive Verständnis dieser >Gelöstheit` am Stereotyp des jüdischen Händlers. Dieser ist für Simmel der geborene Protagonist des sozialen Ausgleichs und der Objektivität, da er weder »an einer räumlichen« noch »an einer ideellen Stelle des gesellschaftlichen Umkreises fixiert ist«. Mit solchen, wenn-

15. Claudia Breger: Ortlosigkeit des Fremden. `Zigeunerinnen< und `Zigeuner in der deutschsprachigen Literatur um 1800, Köln [u.a.]: Böhlau 1998.

16. Sigrid Bauschinger/Horst Denkler/Wilfried Malsch (Hg.): Amerika in der deutschen Literatur. Neue Welt, Nordamerika, USA, Stuttgart: Reclam 1975; Alexander Ritter (Hg.): Deutschlands literarisches Amerikabild. Neue Forschungen zur Amerikarezeption in der deutschen Literatur, Hildesheim, New York: Olms 1977; Manfred Durzak: Das Amerika-Bild in der deutschen Gegenwartsliteratur. Historische Voraussetzungen und aktuelle Beispiele, Stuttgart: Kohlhammer 1979; aus soziologischer Perspektive: Georg Kamphausen: Die Erfindung Amerikas in der Kulturkritik der Generation von 1890, Weilerswist: Velbrück 2002.

17. Hier und im Folgenden: "Georg Simmel: Exkurs über den Fremden«, in: ders.: Soziologie. Untersuchungen über die Form der Vergesellschaftung [1908], Gesamtausgabe, hg. von 0tthein Rammstedt, Bd. 11, Frankfurt/M.: Suhrkamp 1992, S. 764-771. 
gleich positiv umfunktionierten, essenziellen Identitätsbestimmungen schreibt Simmel freilich den Mythos des wandernden Juden Ahasver fort, der zum Kernbestand antisemitischer Klischees gehört. ${ }^{18}$ Ausgeschlagen wird damit einmal mehr die soziale Beschwichtigungsformel der bürgerlichen Assimilation.

Die von Emmanuel Lévinas entwickelte moralphilosophische Konzeption des Anderen ${ }^{19}$ verhält sich gegenüber Identitäts-Zuweisungen analytisch zurückhaltender, obwohl sie in ihrer phänomenologischen Grundierung manche Züge mit Simmels Porträt des Fremden gemein hat. Begriffsgeschichtlich ist >der Andere eine Weiterentwicklung der in Hegels Herr-Knecht-Dialektik entfalteten Problematik wechselseitiger Anerkennung; Lévinas besteht darauf, die Instanz des Anderen als eine black box anzunehmen, die gerade nicht mit konkreten Merkmalsbeschreibungen zu definieren ist. Die starre Verbindung zwischen Menschen und Eigenschaften lösend, weist sein Denken in Richtung einer >Entkörperung` der Kategorie Alterität. Diese Vorstellungen aufgreifend, kann Julia Kristeva schließlich in der bündigen Formel Etrangers à nous-même die interpersonale Relation zwischen dem Ich und seinem Anderen analytisch rückverlagern in die intrapersonale Dynamik der SelbstFremdheit - und eben dadurch trifft sie die politische Dimension der Xenophobie. ${ }^{20}$ Damit verbunden ist ein entscheidender analytischer Perspektivwechsel, der von Personen zu Positionen führt, von den

18. Treffend erfasst hat Simmel bei der engen Verzahnung von Händlertum, Mobilität und Fremdheit, dass manche Elemente des zeitgenössisch aktualisierten Antisemitismus sich gegen Erscheinungen der gesellschaftlichen und kulturellen Moderne als solcher richteten; problematisch allerdings ist seine Tendenz, solche religiösen oder ethnischen Erklärungsmuster positiv zu übernehmen. Wenn völlig unterschiedliche, aber positionshomologe Differenzmerkmale übereinander projiziert werden, führt das zu ihrer Essentialisierung, die den entsprechenden Merkmalsträger zum Fremden schlechthin deklariert. Für neuere soziologische Arbeiten dagegen stellt sich der Fremde als kontingentes Produkt der aufeinander bezogenen Mechanismen sozialer In- und Exklusion dar, wobei die Aufmerksamkeit methodisch nicht auf der jeweils als fremd bestimmten Person oder Gruppe verbleibt, sondern sich jenen Formen der Interaktion und Kommunikation zuwendet, die solchen Zuschreibungen vorausliegen (vgl. R. Stichweh: Der Fremde - Zur Soziologie der Indifferenz, S. $46 \mathrm{ff}$.).

19. Emmanuel Lévinas: Le temps et l'autre (1948), Paris: PUF 1985; ders.: Autrement qu'être au delà de l'essence, Den Haag: Nijhoff 1974; ders.: Die Spur des Anderen. Untersuchungen zur Phänomenologie und Sozialphilosophie, hg. v. Wolfgang Nikolaus Krewani, Freiburg [u.a.]: Karl Alber 1983.

20. Julia Kristeva: Fremde sind wir uns selbst, Frankfurt/M.: Suhrkamp 1990. 
Fremden als distinkter Gruppe mit semantisch stabilen Qualitäten zur Kategorie des Fremden im Sinne eines ebenso sneutralen` wie politisch schlagkräftigen Abstraktums.

Das Fremde, verstanden als ein begriffliches Derivat der zugrundeliegenden Eigenschaft, ist als Kategorie nicht auf bestimmte $\mathrm{Ob}$ jekte oder Akteure gerichtet, sondern auf die Relation, in der sie sich befinden. Fremdheit ist weder eine geographisch noch ethnisch zu fixierende Eigenschaft, sondern eine Beziehungsform ${ }^{21}$ - und als solche stets, implizit oder explizit, Verhandlungssache. Insofern umreißt der Begriff einen durchaus kontroversen und bedeutungsgeladenen Schauplatz sprachlich-symbolischen Handelns. Als Konsens neuerer Arbeiten darf gelten: "Fremdheit 'gibt` es nicht unabhängig von der sprachlichen Bezugnahme auf Fremdheit«. ${ }^{22}$ Dies betrifft die Genese psychologischer Einstellungen ebenso wie das in einer Gesellschaft jeweils für wahr oder richtig gehaltene Wissen über das Fremde und das ästhetische Repertoire seiner Repräsentation. Aus dieser Sicht erscheint es plausibel, im Zeitalter universell zirkulierender Zeichen kultureller Differenz nicht mehr den oder die Fremde/n zum heuristischen Ausgangspunkt zu wählen (was auf der Ebene semantischer Effekte verbliebe), sondern die symbolischen und diskursiven Vorgänge, die ökonomischen, sozialen, institutionellen und medialen Rahmenbedingungen bei der Konstitution des Fremden ins Visier zu nehmen.

Im Verständnis jener Tradition von cultural studies, wie sie u.a. Raymond Williams und Stuart Hall in Birmingham etablierten, bedeuten soziale Prozesse der Hierarchiebildung, In- und Exklusion zugleich eine Dimension kulturellen Handelns und vice versa. So ist verschiedentlich betont worden, dass sich die Herausbildung von Gruppen- oder National-Identitäten im kollektiven Imaginären und mit Hilfe bestimmter Grundmuster des Erzählens vollzieht - Benedict Anderson beschreibt dies mit seinem Konzept der "Imagined Communities «, ${ }^{23}$ Homi Bhabha als programmatischen Zusammen-

21. »Fremdheit ist keine Eigenschaft, auch kein objektives Verhältnis zweier Personen oder Gruppen, sondern die Definition einer Beziehung« (Alois Hahn: „Die soziale Konstruktion des Fremden«, in: Walter M. Sprondel [Hg.]: Die Objektivität der Ordnungen und ihre kommunikative Konstruktion, Frankfurt/M.: Suhrkamp 1994, S. 140-163, hier: S. 140).

22. H. Münkler: Furcht und Faszination, S. 14.

23. Benedict Anderson: Imagined Communities: Reflections on the Origin and Spread of Nationalism, London: Verso 1991; dt.: Die Erfindung der Nation. Zur Karriere eines folgenreichen Konzepts, Frankfurt/M.: Campus 1996. 
hang von Nation und Narration. ${ }^{24}$ Ebenso wirken in der Ausprägung kultureller Differenz symbolische und narrative Anteile mit. ${ }^{25}$ Die alltägliche Durchsetzung des Kolonialismus wäre als Epochenphänomen undenkbar ohne die ihn begleitenden Kulturtechniken der Repräsentation, in den künstlerischen Strömungen des Exotismus beispielsweise, den Manifestationen des Rassismus und der Gewalt. Die koloniale Herrschaft ist als ein Komplex von kulturellen Vorgängen und Mechanismen zu entschlüsseln. ${ }^{26}$ Edward Said hat das Zusammenspiel wissenschaftlicher, kultureller und ästhetischer Praxen in der Genese des Orientalismus exemplarisch analysiert. Was dieser Bewegung des 18. und 19. Jahrhunderts unter dem Namen `Orient` zum Gegenstand wird, ist Produkt und Konstrukt westeuropäischen Denkens. ${ }^{27}$ - Das bringt uns vom Blick auf die Erscheinungsformen der Fremdbilder zu der Frage, wie sie zu einem Gegenstand kulturellen Wissens werden konnten.

\section{Das Wissen des Fremden}

Ethnologie als die Wissenschaft vom kulturell Fremden ${ }^{28}$ formiert sich in Ansätzen bereits in der Antike. ${ }^{29}$ Anders als in die durch mathematisch-geometrische Gesetze vindizierbare Ordnung der physischen Welt fließt in die Berichte über ferne Regionen eine unentwirrbare Mischung aus Phantasie und Hörensagen ein. Was vom Rande der den Griechen vertrauten Ökumene kommt, entzieht sich der rationalen Durchdringung und der empirischen Überprüf-

24. Homi K. Bhabha: Nation and Narration, London [u.a.]: Routledge 1990.

25. Wolfgang Müller-Funk: Die Kultur und ihre Narrative, Wien [u.a.]: Springer 2002.

26. Zur kulturellen Dimension kolonialer Machttechniken Nicholas Thomas: Entangled Objects. Exchange, Material Culture, and Colonialism in the Pacific, Cambridge/Mass [u.a.]: Harvard Univ. Press 1991; ders.: Colonialism's Culture. Anthropology, Travel and Government, Cambridge: Polity Press 1994.

27. Orientalismus ist ein Effekt konvergenter Prozesse der kulturellen und wissenschaftlichen Kolonisierung, die nicht erst auf der Ebene unmittelbarer geographischer Okkupation (Reisen, Erforschen, Erobern) einsetzen, sondern als Haltung und Ausrichtung beginnen, mit der Prägung bestimmter Wahrnehmungs- und Deutungsmuster (Edward W. Said: Orientalismus, Frankfurt/M. [u.a.]: Ullstein 1981).

28. Karl-Heinz Kohl: Ethnologie - die Wissenschaft vom kulturell Fremden, München: Beck 1993.

29. Vgl. Klaus E. Müller: Geschichte der antiken Ethnologie, Reinbek: Rowohlt 1997. 
barkeit. Der 'Kunder von fremden Ländern und Menschen, den Exkursionen in die Ferne der Zeit wie des Raumes gaben Herodots Historien auf eine Weise Gestalt, die noch den (post-)modernen cross-over-Experimenten zwischen Literatur und Wissenschaft zum Vorbild dient. Von sseinem seinem provokativen Vortrag vor der Frobenius-Gesellschaft als einem Zeugen der Grenzüberschreitung von Anthropologie und Poesie. ${ }^{30}$ Am Reisebericht haftet seit der frühen Etablierung dieses Genres etwas Fabelhaftes; die Schilderungen weitentfernter Kulturen sind selbst tangiert von den Abenteuern, denen sie sich verdanken.

$\mathrm{Zu}$ einer strengen, methodisch verfassten Wissensordnung entwickelt sich die ethnologische Praxis vor allem durch die enorme Schubkraft der beiden Entdeckungszeitalter, nach 1500 und um 1750. Zunächst bestimmt ein taxinomisches, archivierendes Sprachwissen, später eher klimageographische und anthropometrische Beobachtungsreihen den Grundbestand ethnologischer Daten. Im Zuge des historistischen Denkens der zweiten Hälfte des 19. Jahrhunderts heftet sich ein genealogisches Erklärungsinteresse an das Studium des Fremden. Die geographisch fernstehenden, ethnisch als 'primitiv taxierten Kulturen sind Residuen aus den Anfangskapiteln der Menschheit; sie halten den Europäern das Bild ihrer phylogenetischen Abkunft vor Augen. Die Kunde vom Fremden wird, ausgerechnet im Zeitalter der Herstellung zuvor ungekannter globaler Synchronie, zur ,Ur-Geschichte`, zum Rückwendungs- und Suchunternehmen nach den Wurzeln und Quellen der Kultur. Anders die religionswissenschaftlichen und psychohistorischen Fragestellungen, die sich etwa in der Auseinandersetzung mit dem Animismus (Frazer, Lévy-Bruhl) herausbilden. ${ }^{31}$ Sie versuchen die Herausforderung der westlichen Moderne durch fremde Kulturen mit erzählenden, komparatistischen Methoden aufzunehmen. Freuds Lektüre dieser Schriften in Totem und Tabu führen ihn zur vergleichenden Entdeckung des Unbewussten im Triebgeschehen seiner Wiener Patienten.

30. Hubert Fichte: »Ketzerische Bemerkungen für eine neue Wissenschaft vom Menschen« (1976), in: Petersilie. Die afroamerikanischen Religionen, Frankfurt/M.: Fischer 1984, S. 359-365.

31. James George Frazer: The Golden Bough. A Study in Magic and Religion, London: Macmillan 1922; dt.: Der goldene Zweig. Das Geheimnis von Glauben und Sitten der Völker, Reinbek: Rowohlt 1989; Lucien Lévy-Bruhl: Les fonctions mentales dans les sociétés inférieures, Paris: Alcan 1910; dt. Das Denken der Naturvölker, Wien: Braumüller 1926. 
Der Rahmen einer anthropologischen, zumal prähistorisch verengten Fachdisziplin ist angesichts wachsenden Reise- und Sammlerfleißes immer weniger in der Lage, die Reichhaltigkeit des ethnologischen Materials zu verarbeiten. Wo ein solcher fachlicher $\mathrm{Zu}$ sammenhalt nicht mehr zu organisieren ist, tritt häufig die Institution selbst als rezeptives Medium in diese Funktion ein: das Museum oder die wissenschaftliche Gesellschaft. Oft vereinigt allein das charismatische Wirken interdisziplinärer Pioniere vom Schlage eines Rudolf Virchow die disparaten Fundstücke, indem es sie dem Zugriff einer Kunstkammer unter hochtechnischen Bedingungen unterwirft - angetrieben von einer Obsession für die Objekte und ihre klassifikatorische Erfassung. Ohnedies hat sich das 19. Jahrhundert mit der Hinwendung zum Positiven und Empirischen einer Epistemologie des Sammelns verschrieben. Die schiere Menge der Sammlerstücke und ihre demonstrative physische Präsenz sollen und müssen für sich selbst sprechen, je weniger durch das Gesamt aller Objekte, Dokumente und Werke noch an historischem Sinngefüge zu gewinnen ist.

Jacob Burckhardt spottete, das 19. Jahrhundert werde einst »das gebildete « heißen. Noch nie war eine Zeit derart wohlinstruiert, im Besitze schönster Traditionsbestände und in Reichweite aller denkbaren und undenkbaren kulturellen Hervorbringungen. Im Positivismus-Taumel der vielerlei Daten und Fakten, der materialisierten Beweise und Belege für immer ungreifbarere Zusammenhänge kann sich die Komplizenschaft zwischen dem Disparaten und dem Fremden auch auf medialem Niveau bewähren. In abenteuerlichen Forschungsreisen durch Asien, Afrika, Lateinamerika bildet sich ein neuer Typus des Jägers und Sammlers heraus. Mit gleicher Hingabe bringen deutsche Gelehrte Schädel und Knochen nach Hause, $\mathrm{Mu}$ sikinstrumente und Kochgeschirr, Bildpostkarten und Anekdoten. Alles Exotische hatte den Reiz der Sensation; der Ausstellungswert eines musealen Schauobjekts und die von ihm entfachte Neugier wuchsen im Maße dessen, was für das Aufspüren, Erlangen und Transportieren an Aufwendungen erbracht worden ist. Bei der heimischen Schaustellung wilder Tiere oder gar fremdkultureller Menschen galt zudem, dass sie, die nun eben keine stillgestellten Objekte waren, sondern artfremd zugerichtete Kreaturen, mit ihrer stummen Anklage dem Beschauer ein leichtes Schaudern einflößen konnten. Das Fremde lässt sich nicht bildungsästhetisch neutralisieren, es blieb auf die Mitwirkung und Vorstellungskraft seines Publikums angewiesen. Fast unmerklich haben sich entlang der künstlerisch und optisch generierten Bildwelten des 19. Jahrhunderts neue 
Formen des Schauens, Betrachtens und Beobachtens entwickelt. ${ }^{32}$ Nach 1900 hat man sich den idealen Rezipienten des Fremden nicht mehr als vorsichtig reisenden Amateur vorzustellen, sondern als versierten Museums- und Ausstellungsbesucher, als passionierten Kolonialwarenkunden und Kinogänger.

Mit seinen subjektiven und medienästhetischen Anteilen widersetzt sich das ethnologische Wissen einer rein sachlichen oder gar systematischen Behandlung. In einer für die gefestigte bürgerliche Sittlichkeit geradezu unerhörten Weise wirken die Fund- und Schaustücke aus überseeischen Kulturen sinnlich aufgeladen. Die Grenze zwischen Wissenschaft und Voyeurismus ist fließend, wie etwa die seit den 1880er Jahren beliebte Zurschaustellung von Menschen mit Ganzkörper-Tätowierungen belegt, bei der sich die gelehrte Lust am Abnormen, Monströsen oder Kuriosen mit einer circensischen Erotik verband. Die strikten Zensurauflagen für die Abbildung entblößter Körper, sie gelten nicht für ethnographische Dokumentationen im Dienste der Wissenschaft. Auch Bildbände über Die Rassenschönheit des Weibes oder Das Weib im Leben der Völker, deren sexualisierte Posen die Grenze des interesselosen Wohlgefallens ganz offensichtlich zu unterlaufen trachten, gehören zum weitgesteckten Gegenstandsrepertoire des kulturellen Wissens vom Fremden. ${ }^{33}$ Nicht nur in solchen Grenzfällen gilt: Der Darstellung als solcher schon wohnt ein sensualistisches surplus inne, dessen sittliche Folgen kaum einzuschätzen und noch schwieriger zu kontrollieren sind. In der affektiven und emotionalen Besetzung des ausgestellten Fremden zeigt sich dessen kulturelle Provokation. Denn beunruhigend ist nicht das schlechthin Andere, sondern dasjenige, was im Habit des Fremden heimlich-unheimliche Resonanzen auslöst.

Die Wechselwirkung von ego und alter trat als das entscheidende Momentum des ethnographischen Blicks hervor, als nach 1900

32. Jonathan Crary: Techniques of the observer (1990); dt.: Techniken des Betrachters. Sehen und Moderne im 19. Jahrhundert, Basel [u.a.]: Verl. der Kunst 1996.

33. Vgl. in den Jahrzehnten um die Jahrhundertwende folgende Titel: Heinrich Ploss: Das Weib in der Natur- und Völkerkunde (1885), hg. v. Max Bartels, 2 Bde., Leipzig: Grieben ${ }^{3} 1891$; Johannes Ranke: Der Mensch (1893), 2 Bde., Leipzig: Bibliogr. Inst. ${ }^{3} 1911 / 12$; Carl Heinrich Stratz: Die Rassenschönheit des Weibes (1901), Stuttgart: Enke ${ }^{5} 1904$; Friedrich Ratzel: Völkerkunde, 2 Bde., Leipzig [u.a.]: Bibliogr. Inst. '1895; Emil Selenka: Der Schmuck des Menschen, Berlin: VITA 1900; Albert Friedenthal: Das Weib im Leben der Völker (1910), 2 Bde., Berlin: Verlagsanst. für Literatur und Kunst 1922; und schließlich: Ferdinand von Reitzenstein: Das Weib bei den Naturvölkern (1923), Berlin: Neufeld \& Henius 1931. 
die Generation der armchair-Ethnologen durch das neue Ethos der Feldforschung abgelöst wurde. Statt Quellen- und Archivstudium ist nun Autopsie gefragt, die aufwändige Reise zu entlegensten Regionen und Ethnien, der längerfristige Aufenthalt 'vor Ort ‘ und möglichst nahe den lebenden Studienobjekten. Das von Bronislaw Malinowski formulierte Prinzip der teilnehmenden Beobachtung stellt den ebenso ingeniösen wie riskanten Versuch dar, aus der Betrachterabhängigkeit des ethnographischen Forschens dessen wissenschaftliches Selbstverständnis zu gewinnen. Als methodische Haltungen sind empathische Teilnahme und distanzierte Beobachtung prinzipiell unvereinbar; im ethnographischen Lebensexperiment ist ihr Konflikt nie auszuräumen, wie Malinowskis spät publik gewordene private Tagebuch-Mitschriften zu den offiziellen Beobachtungs-Protokollen verraten. ${ }^{34}$ Voreinstellungen, Wünsche und Ängste des Ethnologen schreiben mit an den Ergebnissen; seine Texte sind, vergleichbar den Werken der Romanautoren und der Historiker, von rhetorischen und ästhetischen Figuren durchzogen. ${ }^{35}$ Die reflexive Wendung des ethnologischen Wissens, der Weg vom Archiv fremdartiger Sitten und Gebräuche zur Aufmerksamkeit für die Zwischenposition des Beobachters, ist in der amerikanischen >Writing-Culture «-Debatte als methodisches, aber auch als moralisches Problem bedacht worden. ${ }^{36}$

34. Bronislaw Malinowski: Argonauten des westlichen Pazifiks, Frankfurt/M.: Syndikat 1979; ders.: Ein Tagebuch im strikten Sinne des Wortes: Neuguinea 19141918, Frankfurt/M.: Syndikat 1985.

35. Auf dem Gebiet der Geschichtsschreibung hat dies exemplarisch Hayden White: Metahistory. Die historische Einbildungskraft im 19. Jahrhundert in Europa, Frankfurt/M.: Fischer 1991 herausgearbeitet; für die Anthropologie vgl. Marschall Sahlins: Historical Metaphors and Mythical Realities, Ann Arbor: Univ. of Michigan Press 1981; James Fernández (Hg.): Beyond Metaphor. The Theory of Tropes in Anthropology, Stanford: Stanford Univ. Press 1991.

36. James Clifford/George E. Marcus (Hg.): Writing Culture. The Poetics and Politics of Ethnographie, Berkeley: Univ. of California Press 1986; Clifford Geertz: Die künstlichen Wilden. Anthropologen als Schriftsteller, München: Hanser 1990; Johannes Fabian: Time and the other. How anthropology makes its object, New York: Columbia Univ. Press 1983. Einen Überblick zur US-amerikanischen Debatte und ihrer europäischen Rezeption bieten: Eberhard Berg/Martin Fuchs (Hg.): Kultur, soziale Praxis, Text. Die Krise der ethnographischen Repräsentation, Frankfurt/M.: Suhrkamp 1993; Doris Bachmann-Medick (Hg.): Kultur als Text. Die anthropologische Wende der Literaturwissenschaft, Frankfurt/M.: Fischer 1996. Zum Verhältnis zwischen Ethnographie und Literatur aus deutscher Sicht vgl. weiterhin: Doris Bachmann-Medick: Übersetzung als Repräsentation fremder Kulturen, Berlin: Schmidt 1997; Dietrich 
Aus den Aporien der Ethnologie als einer Wissenschaft vom Fremden, die im eigenen Diskurs >die Anderen` zur Sprache kommen lassen will, erwachsen offenbar Motive und Fragestellungen, die eine besondere Affinität zur literarischen Ästhetik erkennen lassen: Erkundungen nach der subjektiven Autorschaft, der erzählerischen Perspektivierung und den Redeformen, nach Fiktionalität und Polysemie, Fragen nach der Projektion und Imagination anlässlich der Begegnung mit dem Fremden, nach der Darstellbarkeit von Gewalt; vor allem aber: Neugier, Erstaunen, Erschrecken. Die >Krise der Repräsentation` haben die aus der Feldforschung berichtenden Ethnologen empirischer erfahren als die am Schreibtisch den Texten nachspürenden Philologen. Schon die als Vorläufer der neuerlich diskutierten Grenzüberschreitung zwischen Wissenschaft und Poesie geltenden Afrika-Reisenden Victor Segalen und Michel Leiris haben die Komplexität der Begegnung mit der Fremde und deren Präsenz im Schreibakt reflektiert. Der zugereiste Forscher teilt nicht nur mit, was er sieht und in Erfahrung bringt, sondern er registriert auch das Echo seiner Präsenz, ${ }^{37}$ den in der Konfrontation sich auswirkenden Transfer der Kulturen.

In diesem Sinne gehören nicht nur Feldstudien unter, wie es bezeichnenderweise heißt, 'wildfremden` Menschen zur ethnographischen Praxis, sondern auch binnenkulturelle Rezeptionsformen und exotistische Surrogate: die Inszenierung des Fremden in Museen und Völkerschauen, die Herstellung und Verbreitung exotischer Ikonographie in Zeitschriften und Reklame, der architektonische Einsatz orientalischer Stilzitate und stropischer Interieurs, die Jagd nach neuartigen ästhetischen Valeurs in Bild- und Wortkunst, nicht zuletzt die philosophische Hinwendung zum pensée sauvage magisch-animistischer Rituale oder zu den Religionen außereuropäischer Hochkulturen. All diese Bestrebungen, die so offensichtlich im Banne des Fremden stehen, betreiben auch, oder sogar überwiegend, Selbsterkundung; sie arbeiten an der Sichtbarkeit von Kultur durch Differenz.

Gerade der prosperierende Bereich des >Fremde-Wissens zeigt: Die Beobachtung kultureller Muster gelingt am ehesten nicht bei der eigenen Kultur, sondern bei jener der anderen. Auf diesem

Harth (Hg.): Fiktion des Fremden. Erkundung kultureller Grenzen in Literatur und Publizistik, Frankfurt/M.: Fischer 1994; Klaus R. Scherpe/Alexander Honold (Hg.): Das Fremde. Reiseerfahrungen, Schreibformen und kulturelles Wissen, (Beiheft Nr. 2 zur Zeitschrift für Germanistik) Bern [u.a.]: Peter Lang Verlag 2000 (2. Auflage 2002).

37. James Clifford: The Predicament of Culture, Cambridge/Mass.: Harvard Univ. Press 1988. 
Umweg wird sichtbar, was den je eigenen Wahrnehmungsgewohnheiten transparent und darum unsichtbar bleibt. Als methodische Innovation ist das Verfremdungspotential des interkulturellen Blicks eng verbunden mit der Herausbildung kulturwissenschaftlicher Arbeitsweisen, die ihren Blick auf kollektive Symbolbestände, Mentalitäten und Praktiken am Außereuropäischen schulen. So reist Aby Warburg 1896 durch das Gebiet der Pueblo-Indianer Neumexikos, um deren Maskentänze als Paradigma heidnischer Religiosität zu studieren; doch sind die Rituale der Indios mehrfach überschrieben durch Schichten spanisch-katholischer und nordamerikanischer Erziehung und müssen gleichsam wie ein literarisches Palimpsest herauspräpariert und entziffert werden. ${ }^{38}$ Auch in Archäologie und Kunstgeschichte wird Alterität zur entscheidenden Signatur der Gegenstände. So hat insbesondere die Ikonographie Erwin Panofskys die konstitutive Leistung der aufgekündigten Sinntransparenz betont. ${ }^{39}$ An Gesten, Posen und weiteren Körpersignalen, die in der europäischen Alltagskultur einen genau definierten pragmatischen Aussagewert haben, lehrt Panofsky das Arbiträre, die kulturelle Konstruktion zu sehen - durch Verfremdung, also den Hinweis auf abweichende Semantisierung dieser Gesten in anderen Kulturen. ${ }^{40}$ Das nur scheinbar naturwüchsige Band zwischen Körpersprache und Bedeutung wird gelockert. Dadurch erst kann >Verkörperung ‘ als kulturelle Zeichenpraxis und performative Dimension in der Kulturanalyse eine Rolle spielen.

Die Wurzeln derartiger Einsichten, die im anthropological turn der Geisteswissenschaften ebenso produktiv werden wie bereits in den ästhetischen Verfremdungs-Schulen der Moderne (etwa des Surrealismus und des Brechtschen Theaters), reichen weit zurück mindestens bis zum Entdecker-Enthusiasmus der Aufklärung und

38. Aby M. Warburg: Schlangenritual. Ein Reisebericht, Berlin: Wagenbach 1988, S. 10.

39. Karl Mannheim (1922): „Beiträge zur Theorie der Weltanschauungs-Interpretation«, in: Wissenssoziologie. Auswahl aus dem Werk, hg. v. Kurt H. Wolff, Neuwied [u.a.]: Luchterhand 1964, S. 91-154; Erwin Panofsky (1939): »Ikonographie und Ikonologie«, in: Ekkehard Kaemmerling (Hg.): Bildende Kunst als Zeichensystem 1: Ikonographie und Ikonologie, Köln: DuMont 1979, S. 207- 225.

40. Panofsky erläutert die kulturelle Bedingtheit solcher Gesten am Beispiel des Hutziehens: »Weder von einem australischen Buschmann noch von einem alten Griechen könnte man die Erkenntnis erwarten, daß das Ziehen des Hutes nicht nur ein praktisches Ereignis mit gewissen ausdruckshaften Nebenbedeutungen ist, sondern auch ein Zeichen der Höflichkeit« (E. Kaemmerling: Ikonographie und Ikonologie, S. 208). 
seiner Ansätze einer reflexiv verfremdeten Selbstbeobachtung, wie sie die Swiftsche Gulliver-Figur oder Montesquieues Lettres persanes unternehmen. Ähnlich wie die camera obscura, und doch mit ganz anderen Mitteln und Absichten, sind die ästhetischen Experimente, die Collagen und Montagen der Moderne darauf aus, etablierte Sehgewohnheiten auf den Kopf zu stellen.

Es ließe sich hier der große Bogen ästhetisch induzierter Lernprozesse einer verfremdenden Selbstaufklärung nachzeichnen, der von Swift bis Brecht, oder von den Indianern Montaignes bis zu jenen Hubert Fichtes in seiner Geschichte der Empfindlichkeit führte. ${ }^{41}$ Ein Lernprozess der fortschreitenden Entzauberung eurozentrischer Illusionen, in dem Poesie und Wissen gleichermaßen und mit je eigenen Mitteln ihre Anteile hatten. Der Auftritt des Fremden als Form der Selbsterkenntnis: Das freilich wäre eine andere Geschichte, die es dann tatsächlich mit einer Umkehrung der Perspektive zu tun bekäme. Denn Rimbauds emphatischer Bekenntnissatz »Ich ist ein anderer", er wartet immer noch auf die ihm einzig korrespondierende Antwort, auf die Einsicht nämlich: Der Andere - jeder andere - ist ein Ich.

41. Hubert Fichte: Die Geschichte der Empfindlichkeit, hg. v. Gisela Lindemann, Frankfurt/M.: S. Fischer $1989 f f$. 